\title{
Concentrating Partial Entanglement by Local Operations
}

\author{
Charles H. Bennett \\ IBM Research Division, T.J. Watson Center, Yorktown Heights, NY 10598, USA. \\ Herbert J. Bernstein \\ Hampshire College, Institute for Science and Interdisciplinary Studies, Amherst, MA, 01002 USA. \\ Sandu Popescu \\ Physics Dept. Tel Aviv University, Israel. \\ Benjamin Schumacher \\ Physics Department, Kenyon College, Gambier, OH 43022, USA.
}

(February 1, 2008)

\begin{abstract}
If two separated observers are supplied with entanglement, in the form of $n$ pairs of particles in identical partly-entangled pure states, one member of each pair being given to each observer; they can, by local actions of each observer, concentrate this entanglement into a smaller number of maximally-entangled pairs of particles, for example EinsteinPodolsky-Rosen singlets, similarly shared between the two observers. The concentration process asymptotically conserves entropy of entanglement - the von Neumann entropy of the partial density matrix seen by either observer - with the yield of singlets approaching, for large $n$, the base-2 entropy of entanglement of the initial partly-entangled pure state. Conversely, any pure or mixed entangled state of two systems can be produced by two classically-communicating separated observers, drawing on a supply of singlets as their sole source of entanglement.
\end{abstract}

PACS numbers: 03.65.Bz, 42.50.Dv, 89.70.+c 


\section{INTRODUCTION}

Recent results in quantum information theory have shed light on the channel resources needed for faithful transmission of quantum states, and the extent to which these resources can be substituted for one another. The fundamental unit of quantum information transmission is the quantum bit or qubit [1]. A qubit is any two-state quantum system, such as a spin-1/2 particle or an arbitrary superposition of two Fock states. If two orthogonal states of the system are used to represent the classical Boolean values 0 and 1, then a qubit differs from a bit in that it can also exist in arbitrary complex superpositions of 0 and 1 , and it can be entangled with other qubits. Schumacher's quantum data compression theorem [1,2] characterizes the number of qubits, sent through the channel from sender to receiver, that are asymptotically necessary and sufficient for faithfully transmitting unknown pure states drawn from an arbitrary known source ensemble.

Quantum superdense coding [3] and quantum teleportation [4] consume a different quantum resource - namely entanglement, in the form of maximally entangled pairs of particles initially shared between sender and receiver - and use it to assist, respectively, in the performance of faithful classical and quantum communication. Following Schumacher's terminology, we define an ebit as the amount of entanglement between a maximally entangled pair of

two-state systems, such as two spin- $\frac{1}{2}$ particles in the singlet state, and we inquire how many ebits are needed for various tasks. In [4], for example, it is shown that the consumption of one shared ebit, together with the transmission of a two-bit classical message, can be substituted for the transmission of one qubit.

An important concept in quantum data transmission is fidelity, the probability that a channel output would pass a test for being the same as the input conducted by someone who knows what the input was. If a pure state $\psi$ sent into a quantum channel emerges as the (in general) mixed state represented by density matrix $W$, the fidelity of transmission is defined as $F=\langle\psi|W| \psi\rangle$. A quantum channel will be considered faithful if in an appropriate limit the expected fidelity of transmission tends to unity. This means that the outputs are almost 
always either identical to the inputs, or else so close that the chance of distinguishing them from the inputs by any quantum meausrement tends to zero.

Note that qubits are a directed channel resource, sent in a particular direction from sender to the receiver; by contrast, ebits are an undirected resource shared between sender and receiver. For example, if you prepare two particles in a singlet state and give me one of them, the result is the same as if I had prepared the particles and given you one of them. Ebits are a weaker resource than qubits, in the sense that transmission of one qubit can, as just described, be used to create one ebit of entanglement; but the sharing of an ebit, or many ebits, does not by itself suffice to transmit an arbitrary state of a 2-state quantum system, or qubit, in either direction. To do that, the ebits must be supplemented by directed classical bits, as in teleportation.

One would naturally like to know whether, in order to be useful for purposes such as teleportation, entanglement must be supplied in the form of maximally entangled pairs. In particular, could partly-entangled pure states, such as pairs of particles in the state

$$
\cos \theta\left|\uparrow_{A}\right\rangle \otimes\left|\downarrow_{B}\right\rangle-\sin \theta\left|\downarrow_{A}\right\rangle \otimes\left|\uparrow_{B}\right\rangle
$$

be used instead, and, if so, how many such pairs would be needed to substitute for one maximally entangled pair? Note that by using the Schmidt decomposition, and absorbing phases into the definitions of the basis states, any entangled state can be represented by a bi-orthogonal expression of this form, with positive real coefficients [5]

$$
\Psi(A, B)=\sum_{i=1}^{d} c_{i}\left|\alpha_{i}\right\rangle \otimes\left|\beta_{i}\right\rangle,
$$

where $\left|\alpha_{1}\right\rangle,\left|\alpha_{2}\right\rangle \ldots\left|\alpha_{d}\right\rangle$ and $\left|\beta_{1}\right\rangle,\left|\beta_{2}\right\rangle \ldots\left|\beta_{d}\right\rangle$ are orthonormal states of subsystems $A$ and $B$ respectively, and the coefficients $c_{i}$ are real and positive. From the viewpoint of either observer, an entangled state appears as a mixed state, described by a density matrix obtained by tracing over the degrees of freedom of the other observer. These density matrices are diagonal in the Schmidt basis:

$$
\rho_{A}=\operatorname{Tr}_{B}|\Psi(A, B)\rangle\left\langle\Psi(A, B)\left|=\sum_{i} c_{i}^{2}\right| \alpha_{i}\right\rangle\left\langle\alpha_{i}\right|
$$


and similarly for $\rho_{B}$.

The entanglement of a partly-entangled pure state can be naturally parameterized by its entropy of entanglement, defined as the von Neumann entropy of either $\rho_{A}$ or $\rho_{B}$, or equivalently as the Shannon entropy of the squares of the Schmidt coefficients.

$$
E=-\operatorname{Tr} \rho_{A} \log _{2} \rho_{A}=-\operatorname{Tr} \rho_{B} \log _{2} \rho_{B}=-\sum_{i} c_{i}^{2} \log _{2} c_{i}^{2}
$$

Without losss of generality we choose the $\alpha$ and $\beta$ bases such that the sequence of Schmidt coefficients $c_{1}, c_{2} \ldots$ is nonincreasing.

The quantity $E$, which we shall henceforth often call simply "entanglement," ranges from zero for a product state (eg $\theta=0)$ to 1 ebit for a maximally entangled pair of two-state particles (eg $\theta=\pi / 4)$. (More generally, a maximally entangled state of two subsystems has $d$ equally weighted terms in its Schmidt decomposition, giving $\log _{2} d$ ebits of entanglement, where $d$ is the Hilbert space dimension of the smaller subsystem.)

If a partly entangled pair, with $E<1$, is used directly for teleportation, unfaithful transmission will result. If it is used for superdense coding, the resulting classical channel will be noisy. In this paper we show how, by local operations on a large number $n$ of identical partly entangled pairs, one can concentrate their entanglement into a smaller number of maximally entangled pairs such as singlets. This process of "entanglement concentration" is asymptotically efficient in the sense that, for large $n$, the yield of singlets approaches $n E-O(\log n)$. Conversely, local operations can be used to prepare arbitrary partly-entangled states $\Psi(A B)$ of two subsystems from a starting material consisting of standard singlets, again in a manner which asymptotically conserves entropy of entanglement.

We should clarify what we mean by local operations. Initially the $n$ partly-entangled pairs are shared between two parties (call them Alice and Bob) with Alice receiving one member of each pair, and Bob receiving the other. This non-local sharing establishes an initial entanglement $n E$ between Alice and Bob. After that Alice and Bob operate locally on their particles, with Alice for example performing unitary operations and von Neumann or generalized measurements in the Hilbert space of her particles, and Bob performing similar 
operations in that of his particles. We allow Alice and Bob to coordinate their actions through exchange of classical messages, but not to exchange any quantum systems nor to perform any nonlocal operation after the initial sharing. This restriction is of course necessary to force Alice and Bob to use the partly entangled pairs they already have, rather than generating perfectly entangled pairs de novo.

\section{ENTANGLEMENT CONCENTRATION}

In this section we describe a method whereby the entanglement present in a supply of identical partially-entangled pairs of 2-state particles can be concentrated into a smaller number of perfect singlets. The generalization to $d>2$ state particles is straightforward. We call the method Schmidt projection because its essential step is a projection of the joint state of $n$ pairs of particles onto a subspace spanned by states having a common Schmidt coefficient.

Let $n$ partly-entangled pairs of 2-state particles be shared between Alice and Bob, so that the initial state is

$$
\Psi(A, B)=\prod_{i=1}^{n}\left(\cos \theta\left|\alpha_{1}(i) \beta_{1}(i)\right\rangle+\sin \theta\left|\alpha_{2}(i) \beta_{2}(i)\right\rangle\right) .
$$

When binomially expanded, this state has $2^{n}$ terms, with only $n+1$ distinct coefficients, $\cos ^{n} \theta, \cos ^{n-1} \theta \sin \theta \ldots \sin ^{n} \theta$. Let one of the parties (say Alice) perform an incomplete von Neumann measurement projecting the initial state into one of $n+1$ orthogonal subspaces corresponding to the power $k=0 \ldots n$ to which $\sin \theta$ appears in the coefficient. Either party can perform this measurement locally, Alice by measuring the particles she has, or Bob by measuring the ones he has. Let Alice perform the measurement, obtaining some outcome $k$. She then tells Bob which outcome she obtained. Alternatively, if Bob and Alice wish not to communicate, Bob can perform his version of the measurement locally, and, by virtue of the original entanglement, he will always obtain the same value of $k$ as Alice has. The probability of outcomes is binomially distributed, with outcome $k$ having probability 


$$
p_{k}=\left(\begin{array}{l}
n \\
k
\end{array}\right)\left(\cos ^{2} \theta\right)^{n-k}\left(\sin ^{2} \theta\right)^{k} .
$$

After some outcome $k$ has been obtained, Alice and Bob will be left with a residual state $\Psi_{k}$ of their spins which is a maximally entangled state in a known $2\left(\begin{array}{l}n \\ k\end{array}\right)$-dimensional subspace of the original $2^{2 n}$ dimensional space. Such states can be used without further ado for faithful teleportation in an $\left(\begin{array}{l}n \\ k\end{array}\right)$-dimensional or smaller Hilbert space; or they can be transformed, as described below, into a standard form such as singlets.

Before describing this optional standardization process, we note that the measurement of $k$ occasionally yields a residual state $\Psi_{k}$ with more entropy of entanglement than the original state $\Psi$. However, neither the measurement of $k$ nor any other local processing by one or both parties can increase the expected entropy of entanglement between Alice's and Bob's subsystems. Consider a measurement or other local treatment applied by Alice, resulting in a classical outcome $j$ and a residual pure state $\Psi_{j}$ of the joint system. This treatement cannot influence the partial density matrix $\rho_{B}$ seen by Bob, since if it did one would have a superluminal communications channel based on Alice's applying or not applying the treatment and Bob measuring $\rho_{B}$. Therefore, depending on the extent of correlation between the residual state and the classical outcomes, the expected entanglement of the residual states lies between $E(\Psi)-H$ and $E(\Psi)$, where $E(\Psi)$ is the original pure state's entanglement and $H=-\sum_{j} p_{j} \log _{2} p_{j}$ is the Shannon entropy of the measurement outcomes. All local treatments (eg generalized or positive operator valued measurements [6]) that Alice might apply can be cast in this form, if necessary by considering her operations to be performed in an appropriately enlarged Hilbert space. In particular, unitary transformations by Alice correspond to one-outcome measurements, which cannot change Bob's partial density matrix $\rho_{B}$ at all, and can only change the eigenvectors, but not the eigenvalues, of Alice's. By the same argument, local actions by Bob cannot increase the expected entanglement between his and Alice's subsystems.

Thus, though Alice and Bob cannot by local actions increase their expected entanglement, they can gamble with it, spending their initial amount on a chance of obtaining a greater 
amount.

We now show how the entanglement in the above perfectly-entangled residual states $\Psi_{k}$ can be efficiently transformed into a standard form such as singlets. Fix some small positive $\epsilon$, with $\epsilon=0$ corresponding to perfect efficiency of transformation. Let the above measurement of $k$ be performed independently on a sequence of batches of $n$ pairs each. Each performance yields another $k$ value; let the resulting sequence of $k$ values be $k_{1}, k_{2}, \ldots k_{m}$, and let

$$
D_{m}=\left(\begin{array}{c}
n \\
k_{1}
\end{array}\right)\left(\begin{array}{c}
n \\
k_{2}
\end{array}\right) \ldots\left(\begin{array}{c}
n \\
k_{m}
\end{array}\right)
$$

be the product of the $\left(\begin{array}{l}n \\ k\end{array}\right)$ values for the first $m$ batches. The sequence is continued until the accumulated product $D_{m}$ slightly lies between $2^{\ell}$ and $2^{\ell}(1+\epsilon)$ for some power $\ell$. For any single-pair entanglement $E$ and any positive $\epsilon$, the probability of failing to come this close to a power of two tends to zero with increasing $m$. Once a suitable $D_{m}$ is found, a local measurement is performed by Alice or Bob or both to project the joint system into one of two orthogonal subspaces, a large space of dimension $2 \cdot 2^{\ell}$ and a relatively small space of dimension $2 \cdot\left(D_{m}-2^{\ell}\right)<\epsilon \cdot 2 \cdot 2^{\ell}$. In the latter case, occurring with probability less than $\epsilon$, a failure has occurred, and all or most of the entanglement will have been lost. In the former case, occurring with probability greater than $1-\epsilon$, the residual state is a maximally entangled state of two $2^{l}$ dimensional subsystems, one held by Alice and one held by Bob. Using the Schmidt decomposition, this can be converted by local unitary operations into a product of $\ell$ standard singlets.

\section{EFFICIENCY}

We deal first with the efficiency of the initial concentration stage, which yields a binomially distributed measurement result $k$ and collapses the initial state of $n$ partly-entangled pairs into a maximally-entangled state between two systems of dimensionality $\left(\begin{array}{l}n \\ k\end{array}\right)$. We show below that its efficiency approaches unity for large $n$. Then we show that the sec- 
ond, or standardization, stage, which distills standard singlets from these high-dimensional maximally-entangled states, also approaches unit efficiency for large $m$.

We adopt the local viewpoint of one of the observers, say Alice. From her viewpoint, the initial state is a mixed state of entropy $n E$. Performing the measurement of $k$ splits off some of this entropy in the form of the entropy of the distribution of outcomes $k$, and leaves the rest of it as concentrated entropy of entanglement between the two residual maximallyentangled $\left(\begin{array}{l}n \\ k\end{array}\right)$-dimensional subsystems. The expected amount of concentrated entropy of entanglement is given by

$$
\sum_{k=1}^{n-1}\left(\cos ^{2} \theta\right)^{n-k}\left(\sin ^{2} \theta\right)^{k}\left(\begin{array}{l}
n \\
k
\end{array}\right) \log _{2}\left(\begin{array}{l}
n \\
k
\end{array}\right) .
$$

Because the entropy of the binomial distribution of $k$ values increases only logarithmically with $n$, the fraction of the original entanglement $n E$ captured as concentrated entanglement approaches 1 in the limit of large $n$.

We now the show that the efficiency of distilling standard singlets from large-dimensional fully-entangled states produced by the measurements of $k$ also tends to unity. In the light of the previous discussion, it suffices to show that for any fixed batch size $n>1$, the sequence

$$
Z_{m}=\bmod \left(\log _{2} D_{m}, 1\right)=\log _{2} D_{m}-\ell
$$

of mantissas of base-2 logarithms of $D_{m}$, where $D_{m}$ is given in equation 7 , has an infimum of 0 . This in turn follows from the fact that the binomial coefficients $\left(\begin{array}{c}n \\ k_{1}\end{array}\right),\left(\begin{array}{c}n \\ k_{2}\end{array}\right) \ldots$ are independently drawn from a fixed distribution (eq 6). The evolution of $Z_{m}$ with increasing $m$ may therefore be viewed as a random walk on the unit interval (with wraparound), starting at the origin and taking steps of sizes $\bmod \left(\log _{2}\left(\begin{array}{l}n \\ k\end{array}\right), 1\right)$ and probabilities given by equation 6. It is elementary to show that for any distribution of step sizes, and any positive $\epsilon$, such a walk visits the interval $\left[0, \log _{2}(1+\epsilon)\right]$ with probability 1 , from which the theorem follows.

The Schmidt projection method of entanglement concentration requires at least $n=2$ partly-entangled pairs, and only becomes efficient for large $n$. We now describe another method that works, albeit inefficiently, even with a single partly entangled pair, as in eq. 1 . 
We call this procedure the Procrustean method of entanglement concentration, because its goal is to cut off and discard the extra probability of the larger term in equation 1, leaving a perfectly entangled state. Assume for the moment that $\theta<\pi / 4$ so that if Alice measures particle 1 in the up/down basis, the up outcome is more likely. Instead of performing this von Neumann measurement, she passes her particle through a polarization-dependent absorber, or a polarization-dependent-reflector (eg, for light, a Brewster window), which has no effect on down spins but absorbs, or deflects into a different beam, a fraction $\tan ^{2} \theta$ of the up spins. If the particle is absorbed or deflected, it is rejected; otherwise it is kept. This treatment does not correspond to any von Neumann measurement in the original 2-dimensional spin space, but rather to a two-outcome generalized measurement or POVM (positive-operator-valued measurement) [6.7]. If the particle is not absorbed or deflected, its residual state after this treatment will be a maximally mixed state of spin up and spin down. Now suppose Alice tells Bob the result of her generalized measurement, and suppose that he does not measure his particle at all, but simply discards it if Alice has discarded hers. The result will be a perfectly entangled state of two particles. The Procrustean method is especially suitable for the type of gambling mentioned earlier: when it works, it always yields more entanglement than the parties started out with.

Both the Schmidt projection and the Procrustean method can be generalized to work on larger Hilbert spaces. Like von Neumann's method for obtaining unbiased random bits from a coin of unknown but time-independent head/tail ratio (setting $\mathrm{HT}=1, \mathrm{TH}=0$, and $\mathrm{TT}=\mathrm{HH}=$ do over , Schmidt projection works even when Alice and Bob do not know how entangled their partly-entangled pairs are, provided all $n$ pairs have equal biases $\theta$. The Procrustean method, on the other hand, requires the bias to be known in advance.

Figure 1 plots the yield of perfectly entangled pairs as a function of $\cos ^{2} \theta$ obtained by the Schmidt projection method with $n=2,4,8$, and 32 (lower 4 curves), in comparison with ideal asymptotic yield $n E=n H_{2}\left(\cos ^{2} \theta\right)$, (top curve) and the yield from the Procrustean method (inverted-V shaped curve). Note that for $n<5$ Schmidt projection is absolutely less efficient than the Procrustean method. 


\section{RELATION OF ENTANGLEMENT CONCENTRATION TO QUANTUM DATA COMPRESSION}

Like the Schmidt projection method of entanglement concentration described above, the technique of quantum data compression [2,1] involves projecting the state of a highdimensional system onto a set of orthogonal subspaces depending on eigenvectors and eigenvalues of an associated density matrix. However, the goals and means of the two techniques are sufficientlly different that neither can be substituted for the other. Indeed, certain quantum data transmission tasks can only be accomplished efficiently by using the two techniques together.

Quantum data compression (QDC) has the goal of encoding an unknown sequence of signals from a known quantum source - ie an ensemble of pure states $\left\{\psi_{j}\right\}$ emitted with specified probabilities $\left\{p_{j}\right\}$-into a smaller Hilbert space than it originally occupies, while introducing negligible distortion. QDC is useful when the source has less than maximal entropy (permitting it to be compressed at all) and consists of non-orthogonal states $\psi_{j}$, necessitating the use of quantum operations to do the compression.

As has previously been noted [1, 8, 12], a quantum source is not fully specified by its density matrix $\rho=\sum_{j} p_{j}\left|\psi_{j}\right\rangle\left\langle\psi_{j}\right|$. By the same token, it is also not fully specified by giving an entangled state $\Psi_{A B}$ of which its density matrix is the partial trace, eg $\rho=\rho_{A}=$ $\operatorname{Tr}_{B}\left|\Psi_{A B}\right\rangle\left\langle\Psi_{A B}\right|$. A quantum source can, however, be fully specified by giving both such an entangled state $\Psi_{A B}$ and a von Neumann or generalized measurement to be performed by Bob, who holds subsystem $B$. This is done in such a way that each of Bob's possible measurement outcomes projects Alice's subsystem into one of the states $\psi_{j}$, and the outcomes occur with the required probabilities $\left\{p_{j}\right\}$. Then each of Bob's measurements tells him which state Alice received from the source at that instant. For example, depending on the measurement performed by Bob, the entangled state of equation 1 can be used to generate either of the following two sources for Alice, one classical, the other distinctively quantum.

- Source $Q$, consisting of orthogonal states $|\uparrow\rangle$ and $|\downarrow\rangle$ emitted with unequal probabili- 
ties $\cos ^{2} \theta$ and $\sin ^{2} \theta$, respectively; and

- Source $Q^{\prime}$ consisting of non-orthogonal states $\psi^{0}=(\cos \theta|\uparrow\rangle+\sin \theta|\downarrow\rangle)$ and $\psi^{1}=$ $(\cos \theta|\uparrow\rangle-\sin \theta|\downarrow\rangle)$ emitted with equal probabilities.

The first source, $Q$, is purely classical in the sense that it could be faithfully compressed by making a complete von Neumann measurement in the up/down basis, and applying conventional data compression (eg Huffman coding) to the resulting classical data.

Although the other source, $Q^{\prime}$, would yield statistically similar data when measured in the up/down basis, the resulting data would be useless for reliably encoding a sequence of $n$ states from the source, because the data would be utterly uncorrelated with which of the $2^{n}$ equiprobable non-orthogonal spin sequences the source had emitted.

At this point some notation is helpful. Let $x$ denote an arbitrary $n$-bit sequence, where $n>2$, and let $\Psi^{x}$ denote the $n$-spin product state resulting when source $Q^{\prime}$ emits a sequence of states indexed by the bits of $x$. For example, taking $x=011$, we have $\Psi^{011}=\psi^{0} \otimes \psi^{1} \otimes \psi^{1}$.

In order to transmit such sequences faithfully and economically, one uses quantum data compression [2,1]. This consists of performing a very gentle, incomplete measurement on the the joint state $\Psi^{x}$ of the spins, which projects the state into one of two complementary subspaces :

- a "likely" subspace of dimensionality $2^{n(H(\rho)+\delta)}$ spanned by the eigenvectors of the largest eigenvalues of the joint density matrix $\rho^{(n)}$, defined as the tensor product of $n$ copies of $\rho$.

- the "unlikely" subspace spanned by the remaining eigenvectors.

If the joint state projects into the likely subspace, one transmits the resulting projected state; if the projection fails, one transmits an arbitrary state. Using $W^{x}$ to denote the (slightly mixed) state resulting from applying quantum data compression to a source sequence $\Psi^{x}$ from the source $Q^{\prime}$, it can be shown that for any positive $\delta$ and $\epsilon$ there exists an $n_{0}$ such that for all $n>n_{0}$, the fidelity of the quantum data compression, ie the probability 


$$
F=\sum_{x} p(x)\left\langle\Psi^{x}\left|W^{x}\right| \Psi^{x}\right\rangle
$$

that its output would pass a test for being the same as the input sequence $\Psi^{x}$, conducted by someone who knew what the input sequence was, is greater than $1-\epsilon$. Infidelity can be thought of as resulting from two causes: failure to project into the likely subspace, and failure of even a successful projection into that subspace to agree with the original state when subsequently tested. Both kinds of infidelity become negligible in the limit of large $n$ 円.

Because a less-than-maximally entangled pure state appears as a less-than-maximally random mixed state to its two separate observers, one could imagine using quantum data compression as an approximate means of entanglement concentration. In other words, by separately compressing their respective subsystems, Alice and Bob could squeeze the original entanglement into a smaller number of shared pairs of qubits. Applying this two-sided compression to $n$ shared pairs of entanglement $E$, Alice's and Bob's projections into the likely subspace would be isomorphic (this can be seen by considering the Schmidt representation of the original entangled state), and the result would be to leave each observer with slightly more than $n E$ qubits having slightly less than $n E$ bits entropy of entanglement with an equal number of qubits held by the other observer. However, the entangled sates produced by such two-sided quantum data compression are never maximally entangled, and in an important sense they become poorer approximations of maximally-entangled states as $n$ increases. A perfectly-entangled state must have all its Schmidt coefficients equal, but the compressed bipartite state $\Psi_{c}(A, B)$, resulting from applying two-sided quantum data

\footnotetext{
${ }^{1}$ Although one is usually more interested in data compression, it is also possible to perform classical or quantum data expansion, in other words to encode the output of a nonredundant source into a larger number of redundant bits or qubits. For example, all states of two qubits can be encoded into the majority-up subspace of three qubits, each of which has a single-particle density matrix with eigenvalues $3 / 4$ and $1 / 4$ and entropy of approximately 0.811 bits.
} 
compression to a state such as the $\Psi(A, B)$ of eq. 5 , describing a set of $n$ partly-entangled pairs, has a distribution of values of Schmidt coefficents whose variance increases with $n$. In consequence, the fidelity with which $\Psi_{c}(A, B)$ approximates any maximally entangled state approaches zero with increasing $n$, as does the fidelity with which it would work in teleporting a random state in a Hilbert space of dimensionality equal to that of one of its two parts.

We have just shown that two-sided quantum data compression does not work as a method of entanglement conentration. Conversely, because any mixed state can be regarded as the partial trace of an appropriate entangled state, one can imagine attempting to use the Schmidt projection method of entanglement concentration in a one-sided manner as a way of performing quantum data compression. As we will show presently, this also does not work. To use Schmidt projection in a one-sided manner would mean making a more aggressive projection than that used in conventional quantum data compression, into subspaces spanned by each distinct eigenvalue of $\rho^{(n)}$, rather than into a single subspace spanned by all the likely eigenvalues and a residual unlikely subspace. However, this projection is too aggressive for the purposes of reliable data transmission. Because the entropy of the distribution of the eigenvalues increases absolutely with $n$, (although it decreases as a fraction of $n$ ), the fidelity of transmission of typical sequences from a source such as $Q^{\prime}$ tends to zero with increasing $n$.

In more detail, the proposed Schmidt projection method of quantum data-compression, whose fidelity we seek to refute, corresponds in the case of the source $Q^{\prime}$ to an incomplete measurement, in which one observes the number $k$ of down spins in an $n$-spin block, leaving a residual quantum state equal to the renormalized projection of the original source sequence into the subspace corresponding to the measurement outcome. We shall show that this coding cannot transmit sequences from the source $Q^{\prime}$ with asymptotically perfect fidelity, even if the measurement outcome $k$ is made available, as classical information, to help in the decoding process at the receiving end of the channel. Such a dual classical/quantum channel setup is certainly no weaker than a purely quantum channel (where the classical outcome 
$k$ would simply be discarded) and is analogous to the dual channel used in teleportation, in which the quantum channel output is postprocessed in a way depending on classical information generated at the sending end. The inability of even this strong kind of Schmidt coding to approach perfect fidelity for source $Q^{\prime}$ follows from the fact, to be demonstrated presently, that with significant probability it maps distinct input sequences onto the same output state.

Recalling notation introduced earlier, we use $\Psi^{x}$ to denote the $n$-spin product state resulting when source $Q^{\prime}$ emits a sequence of states indexed by the bits of bit string $x$. For example, taking $x=011$, we have $\Psi^{011}=\psi^{0} \otimes \psi^{1} \otimes \psi^{1}$. Let $\bar{x}$ denote the Boolean complement of $x$. In our example $\bar{x}$ would be 100 , and correspondingly $\Psi^{\bar{x}}$ would be $\Psi^{100}=\psi^{1} \otimes \psi^{0} \otimes \psi^{0}$. By expanding the states $\Psi^{x}$ and $\Psi^{\bar{x}}$ in the up/down basis, and grouping terms according to $k$, it can readily be seen that the states $\Psi^{x}$ and $\Psi^{\bar{x}}$ differ only by a uniform sign change in all terms of odd $k$. This difference is obliterated by the Schmidt encoding process, which by measuring $k$ randomizes the relative phases of terms of differing $k$. Thus, for any $x$, the encoding process, even if the classical outcome $k$ is made available at the receiving end, maps input states $\Psi^{x}$ and $\Psi^{\bar{x}}$ onto the same output state or distribution of output states. This precludes the achievement of asymptotically perfect fidelity, which by definition requires that with probability approaching unity a typical channel input be mapped onto a pure or mixed output state arbitrarily close to the input in Hilbert space. Here, on the contrary, we have distinct inputs $\Psi^{x}$ and $\Psi^{\bar{x}}$, which for large $n$ are nearly orthogonal, being mapped onto the same output.

In summary, two-sided quantum data compression is too faithful to the original nonmaximally-entangled state to provide good entanglement concentration (whose goal is a maximally entangled state rather than a good approximation to the initial state). Conversely, entanglement concentration by the Schmidt projection method necessarily sacrifices fidelity to the original state in order to produce a maximally entangled output.

Despite these differences, the two techniques can sometimes be fruitfully combined. Suppose Alice has a long sequence of $n$ spins from a nonrandom quantum source such as $Q^{\prime}$ which 
she wishes to faithfully teleport to Bob with a minimal usage of engtanglement. Suppose further that the entanglement Alice and Bob have at their disposal for teleportation purposes is supplied not in the form of standard singlets but rather as pure but less-than-maximally entangled states $\psi_{A B}$. An economical procedure would then be

- use quantum data compression to compress the input sequence to a bulk of slightly greater than $n H\left(\rho\left(Q^{\prime}\right)\right)$ spins.

- use entanglement concentration to prepare standard singlets from the supply of imperfectly entangled pairs.

- teleport each compressed spin using one of the standard singlets.

\section{DISCUSSION}

We have shown that the entanglement in any pure state of a bipartite system can be concentrated by local operations and classical communication into maximally entangled states such as singlets. Here we note that, conversely, an arbitrary partly-entangled state $\Psi(A, B)$ of a bipartite system can be prepared by local operations and classical communication using standard singlets as the only source of entanglement. One way to do this is for Alice first to prepare a copy $\Psi(A, C)$ of the entangled state she wishes to share with Bob, using two systems in her laboratory, $A$ and $C$. Here $A$ is the system she wishes to entangle with Bob's system $B$, and $C$ is a system similar to $B$ but located in Alice's laboratory instead of Bob's. Because $A$ and $C$ are in the same location, $\Psi(A, C)$ can be prepared by purely local operations. Next Alice uses a supply of standard singlets, in conjunction with classical communication, to teleport the state of her local system $C$ into Bob's system $B$. This has the effect [4] of destroying the entanglement between $A$ and $C$ while creating the desired entangled state $\Psi(A, B)$ shared remotely between Alice and Bob. The teleportation consumes $\log _{2} d$ singlets and requires $2 \log _{2} d$ classical bits of communication, where $d$ is the dimen-

sion of the Hilbert space of system $B$, regardless of the entanglement $E$ of the state being 
teleported, which is rather inefficient if $E$ is small. To reduce the consumption of singlets toward the theoretical minimum, Alice need only use Schumacher coding to compress her $C$ systems before teleporting them to Bob, who then applies the inverse decoding operation to re-expand the teleported $B$ systems to their original dimensions.

Because entropy of entanglement can be thus efficiently concentrated and diluted, its positive-definiteness and the nonincrease of its expecation under local operations represent the only limitation on the asymptotic ability of two separated observers to prepare a final pure state $\Psi_{A B}^{\prime}$ given an inital one, $\Psi_{A B}$, by local operations and classical communication. Thus if $\Psi$ and $\Psi^{\prime}$ have equal entanglement, they can be interconverted with efficiency approaching unity in the limit of large $n$; if they have unequal entanglement, the asymptotic yield is $E\left(\Psi^{\prime}\right) / E(\Psi)$. On the other hand, if the initial state is unentangled, the positivedefiniteness of entanglement and the nonincrease of its expectation under local operations together imply that there is no way of locally preparing an entangled final state $\Psi^{\prime}$, even with low yield.

We have argued that entropy of entanglement is a good entanglement measure for pure states because local operations cannot increase its expectation, but can, with asympotically perfect efficiency, interconvert states of equal entropy of entanglement. No similarly simple entanglement measure is known for mixed states. For a mixed state, the subsystem entropies $H_{A}$ and $H_{B}$ need not be equal, and neither they nor any other simple function of $H_{A}, H_{B}$, and the total entropy $H_{A B}$ can be clearly identified with the degree of entanglement. For example, entropies of $H_{A}=H_{B}=H_{A B}=2$ can be realized by two very different mixed states:

- two independent pairs of classically correlated spins, or

- one singlet and a pair of uncorrelated spins.

The former mixed state is naturally regarded as unentangled, since it can be made by mixing unentangled pure states; the latter should be regarded as entangled, since it can be converted into a maximally-entangled state by discarding the second pair of spins. 
Two possible measures of entanglement for the mixed bipartite state $\rho_{A B}$, each of which reduces to entropy of entanglement when $\rho_{A B}$ is pure, are:

- "Entanglement of formation" defined as the least number of shared singlets asymptotically required to prepare $\rho_{A B}$ by local operations and classical communication.

- "Distillable entanglement" defined as the greatest number of pure singlets that can asymptotically be prepared from $\rho_{A B}$ by local operations and classical communication.

These entanglement measures have the desirable feature that their expectations cannot be increased by local operations, but the disadvantage of being hard to evaluate in particular cases because of the implied optimizations over local procedures.

Since any mixed state can be regarded as a pure state over a larger, and partly unseen, Hilbert space, it might seem more elegant (cf [9]) to define the entanglement of a bipartite mixed state $\rho_{A B}$ as the minimum entanglement of any pure state $\Psi(A B C D)$ of a fourpart system, from which $\rho_{A B}$ can be obtained by tracing over states of $C$ and $D$, and where the entanglement of $\Psi(A B C D)$ is computed by partitioning it into subsystems $A C$ and $B D$. Unfortunately, this prescription is unsatisfactory because it would assign positive entanglement to classically correlated mixed states such as $\rho=\frac{1}{2}(|\uparrow \uparrow\rangle\langle\uparrow \uparrow|+| \downarrow \downarrow\rangle \backslash \downarrow \downarrow \mid)$, which can be generated from unentangled initial conditions by local actions (random choice) and exchange of classical messages.

A nontrivial example of an entangled mixed state is provided by the Werner state [10], whose density matrix is

$$
W=\frac{1}{8} I+\frac{1}{2}\left|\Psi^{-}\right\rangle \Psi^{-} \mid
$$

where $I$ is the $4 \times 4$ identity matrix and $\Psi^{-}$is a singlet. This state may be viewed as a 50/50 mixture of totally mixed states (density matrix $\frac{1}{4} I$ ) and singlets; and indeed, since totally mixed states can be manufactured by purely local means, this description constitutes a local probabilistic procedure for generating $n$ Werner states from an expected $n / 2$ singlets. Recently it has been shown [11] that the Werner state can be constructed much 
more economically, as a mixture of pure states each containing only about 0.1176 ebits of entanglement. Moreover, a small yield $\geq 0.0001258$ of arbitrarily pure singlets can be distilled from Werner states by local actions and classical communication [11]. Thus impure Werner states can be both created from and converted into pure singlets. This two-way conversion

is a rough mixed-state analog of the processes of pure-state entanglement concentration and dilution which have been the principal subject of the present paper. However, it is not known whether the interconversion is asymptotically reversible for mixed states, as it is for pure states. In other words, it is not known whether the Werner state's distillable entanglement is equal to, or far less than, its entanglement of formation.

\section{ACKNOWLEDGMENTS}

We wish to thank William Wootters for advice on Schmidt decompositions and many other subjects, and John Smolin for pointing out how to use teleportation to prepare arbitrary entangled states. 


\section{REFERENCES}

[1] B. Schumacher, "On Quantum Coding," Phys. Rev. A (to appear 1995).

[2] R. Jozsa and B. Schumacher, J. Modern Optics 41, 2343-2349 (1994).

[3] C. H. Bennett and S. J. Wiesner, Phys. Rev. Lett. 69, 2881 (1992).

[4] C. H. Bennett, G. Brassard, C. Crépeau, R. Jozsa, A. Peres, and W. K. Wootters, Phys. Rev. Lett. 70, 1895 (1993).

[5] A. Peres, Quantum Theory: Concepts and Methods (Kluwer, 1993) page 131-133.

[6] C.W. Helstrom, Quantum Detection and Estimation Theory (Academic Press, NewYork, 1976), pp.74-83.

[7] N. Gisin (private communication 1993) noted that POVMs can be simply implemented in polarization space by using a polarization-dependent absorber.

[8] C. H. Bennett, G. Brassard, R. Jozsa, D. Mayers, A. Peres, B. Schumacher, and W. K. Wootters, J. Mod. Optics 41, 2307-2314, (1994).

[9] R. Jozsa, J. Mod. Optics 41, 2315-2323 (1994).

[10] R. F. Werner, Phys. Rev. A 40, 4227 (1989), S. Popescu, Phys. Rev. Lett. 72, 797 (1994).

[11] C.H. Bennett, G. Brassard, S. Popescu, J. Smolin and W.K. Wootters, "Purification of Noisy Entanglement and Faithful Teleportation via Noisy Channels" submitted to Phys. Rev. Lett. (1995).

[12] G.Jaeger, A. Shimony, and L. Vaidman, Phys. Rev. A 51, 54-67 (1995). 


\section{FIGURE CAPTION}

1. Yield of maximally-entangled output states from partly-entangled input states ( $\cos \theta \uparrow \downarrow-\sin \theta \downarrow \uparrow)$, as a function of $\cos ^{2} \theta$. Highest curve is entropy of entanglement of input state, equal to the asymptotic yield of the Schmidt projection method. Successively lower smooth curves give yields of Schmidt projection applied to $n=32$, 8, 4 , and 2 input pairs. The $\Lambda$-shaped curve gives yield by the Procrustean method applied to one input pair. 


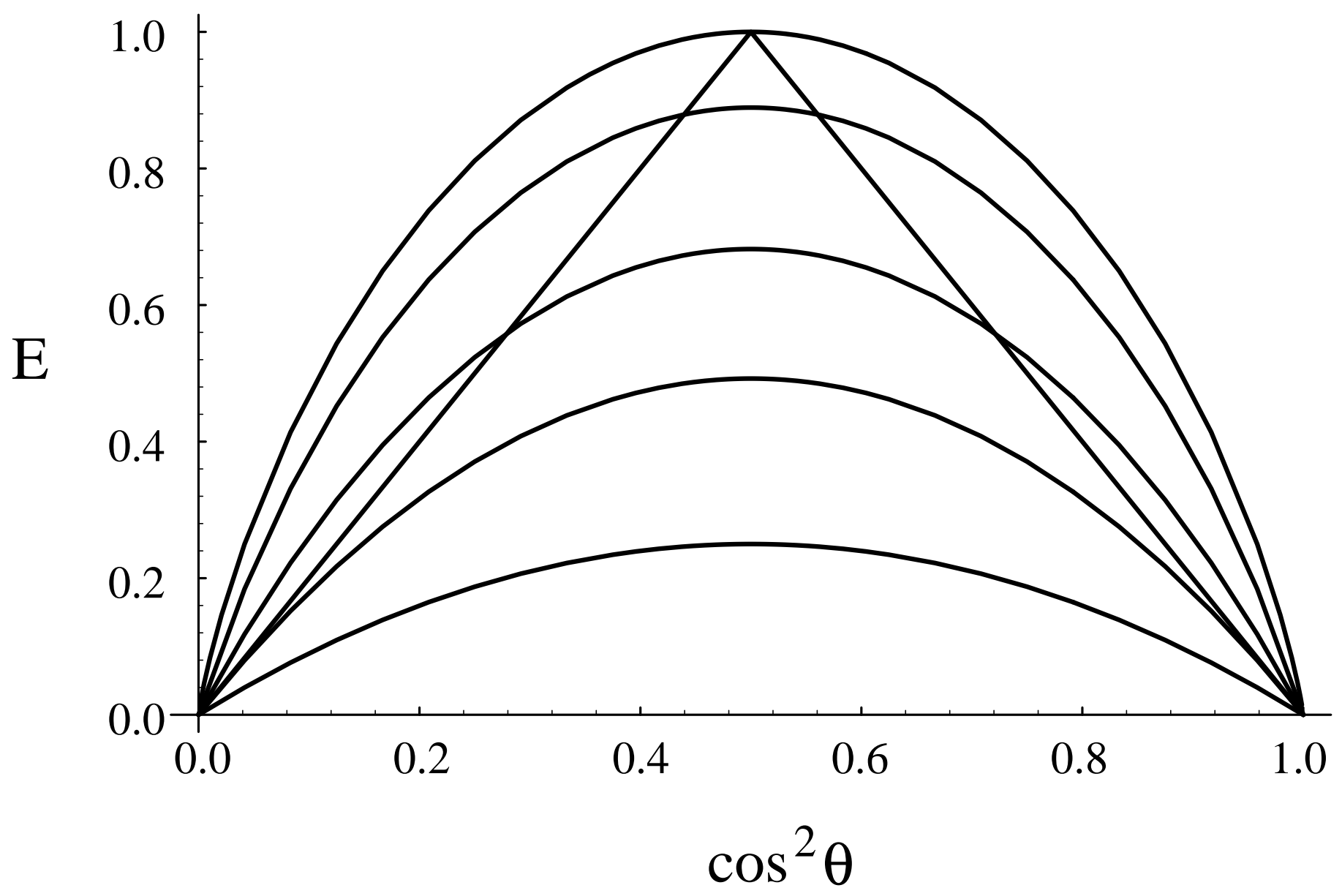

